\title{
Numerical simulation of observations with GOLF on board SOHO
}

\author{
R.A. García ${ }^{\star}$, T. Roca Cortés, and C. Régulo \\ Instituto de Astrofísica de Canarias, E-38205 La Laguna, Tenerife, Spain \\ Received April 11; accepted May 20, 1997
}

\begin{abstract}
The main objective of the GOLF Experiment (Global Oscillations at Low Frequencies) on-board the SOHO (Solar and Heliospheric Observatory) space mission is the quantitative knowledge of the internal structure of the Sun by measuring the spectrum of its global oscillations in a wide frequency range $(30 \mathrm{nHz}$ to $6 \mathrm{mHz})$. There is special interest in detecting the low $\ell \mathrm{p}$ - and g-modes (low frequency modes) which penetrate deeply down into the solar core. The instrument chosen is an improved disk-integrated sunlight resonant scattering spectrophotometer. It obtains the line of sight velocity of the integrated visible solar surface by measuring the Doppler shift of the sodium doublet. Mainly, two innovations have been incorporated to standard earth-based similar apparatus (those from the networks IRIS and BISON). First, GOLF samples each line of the sodium doublet in principle at four points on its wings, using an extra small modulated magnetic field. This new information enables an instantaneous calibration of the measured signal and also opens the possibility to correct from the background solar velocity noise. Second, the use of an extra fixed quarter wave plate, placed at the entrance of the instrument, enables a selection of the circularly polarized solar light. Therefore, the disk averaged solar line-of-sight component of the magnetic field can also be obtained. This is considered as a secondary objective of the mission. In order to study the new information available due to these improvements in the apparatus, the necessity of fully understanding it and the need to write the appropriate software to analyze the data, a complete numerical simulation of the experiment has been built. Running the simulation has yielded two series of 12 months long each, one corresponding to a year of maximum solar activity and the other to a year of minimum solar activity. In this paper the numerical simulation of the GOLF experiment is presented, its sensitivity and instrumental response calculated and a power spectra of this two series have been obtained to show its
\end{abstract}

Send offprint requests to: rgarcia@discovery.saclay.cea.fr

* Present address: CEA/DSN/DAPNIA, SAp, CE Saclay, 91191 Gif-sur-Yvette, France performance against the observations. It is shown that the numerical simulation explains the observed spectra of the solar background velocity signal and the oscillations to a high degree of accuracy.

Key words: Sun: oscillations — methods: data analysis

\section{Introduction}

The SOHO mission consists of a three axis stabilized spacecraft with eleven scientific instruments that studies the Sun (Domingo \& Poland 1988). It offers an unprecedented opportunity to study the deep interior of the Sun through Helioseismology under ideal conditions at the Lagrange $L_{1}$ point. Here, no terrestrial atmospheric effects are present, continuous exposures to the Sun are possible and there is a low Sun-spacecraft line-of-sight velocity. On this spacecraft there are three Helioseismic instruments: GOLF, MDI and VIRGO. Their first objective is the determination of the internal structure and dynamics of the Sun. In particular, GOLF measures the disk integrated radial velocity of the Sun in a frequency range of $10^{-7}$ to $610^{-3} \mathrm{~Hz}$. It is specially designed to detect the low frequency waves that correspond to the low order p-modes (below $2 \mathrm{mHz}$ ) and the g-modes with a sensitivity better than $1 \mathrm{~mm} / \mathrm{s}$ in 20 days and a frequency resolution better than $16 \mathrm{nHz}$ in a two years mission. These modes have not already been detected from the Earth due, mainly, to a combination of two effects: an increase of the atmospheric solar and terrestrial noise at low frequencies and a very low amplitude expected for them. As a secondary objective, GOLF also measures the longitudinal component of the line-of-sight global magnetic field of the Sun with an accuracy better than 1 mGauss.

The instrument is a disk integrated sunlight resonant scattering spectrophotometer. This is an earth-based proved technique that measures the Doppler shift of the solar sodium $D$ lines $(\lambda 5896$ and $\lambda 5890 \AA)$ due to a non zero relative Sun-instrument line-of-sight velocity. 
This technique has been successfully used in solar physics by several researchers (Snider 1970; Brookes et al. 1978a; Fossat \& Roddier 1971). The latest two groups have pioneered the work on helioseismology and, nowadays, the instruments used by helioseismic networks such as IRIS and BISON are based, with some differences, on this kind of technique.

Briefly, the solar absorption line (half-width $\sim 500 \mathrm{~m} \AA$ ) traverses through a sodium vapor cell, placed in a longitudinal magnetic field of 5000 Gauss, which has an intrinsic (thermal) absorption line-width of the order of $25 \mathrm{~m} \AA$. The light is absorbed and reemitted in all directions. This scattered light is symmetrically split into its Zeeman components displaced by $\pm 106 \mathrm{~m} \AA$ from the rest wavelength ( $D_{1}$ case), allowing a measurement on either side on the wings of the solar absorption profile (see Fig. 1). Switching between both wings, by moving appropriately a quarter wave plate and a linear polarizer, alternates the measurement of the intensity on them and therefore, measuring its Doppler shift. The observed velocity is proportional to a normalized difference of intensities that measures the shift.

Compared to the earth-based ones, GOLF incorporates two important improvements. The first one is the possibility of sampling the solar absorption profile at four points by adding a modulating magnetic field of \pm 100 Gauss. Therefore, it can measure the slopes on each wing enabling an instantaneous calibration of the instrument's sensitivity (Isaak \& Jones 1988). The second one is the ability to measure the longitudinal component of the lineof-sight global magnetic field of the Sun by adding a fixed quarter wave plate in front of the other two polarizing elements of the experiment. A full description of the instrument used is to be found in Gabriel et al. (1995). Even though this is a known technique, many items of the existing instruments have never been clearly quantified and correctly understood, as it can be seen from the description of earlier versions of such spectrophotometers (Brookes et al. 1978a; Grec et al. 1991).

Due to the fact of the four sampling points as opposed to the classical two for the earth-based instruments, several definitions of velocity signals can be extracted. Some new parameters can be derived from this new information as the measures of the integrated profile slopes or the new magnetic ratio. For the GOLF spectrophotometer, a quantitative description of the physics of the instrument is to be found in Boumier (1991) and Boumier \& Damé (1993). A scientific breadboard of this kind of spectrophotometer was built and tested with the real Sun (Boumier et al. 1994). Although some new information could be gained from this experiment, the obvious nonidentical working conditions prevented firm conclusions regarding GOLF instrument working at $L_{1}$. As the final design of the GOLF instrument has not been tested with the Sun before launch, a full numerical simulation became a necessity.
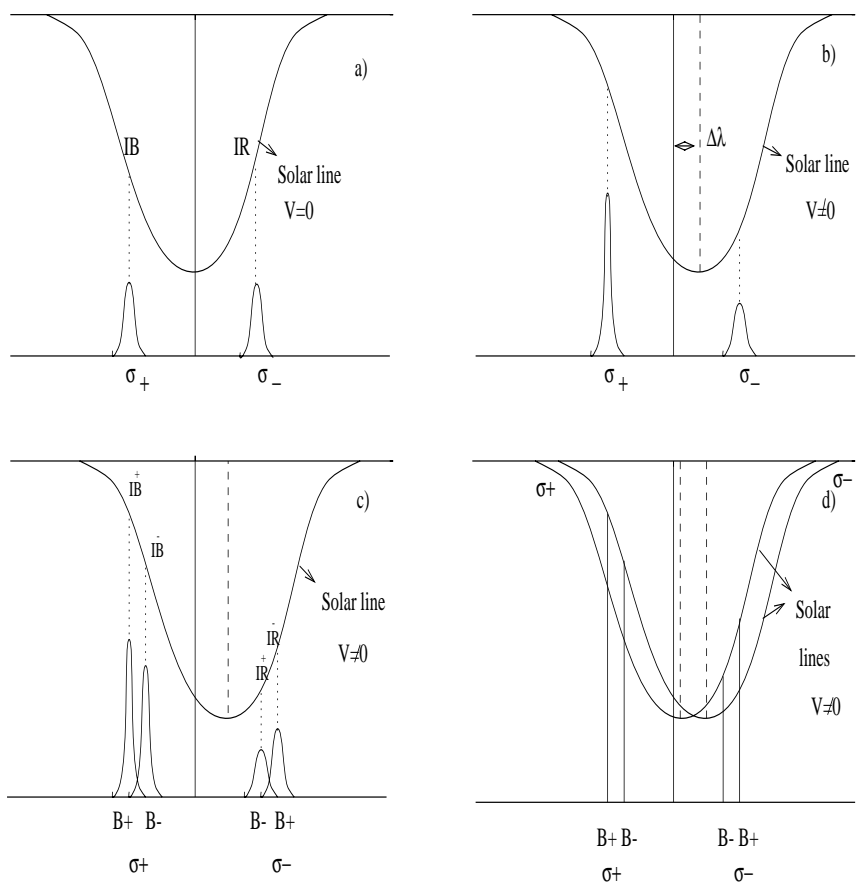

Fig. 1. Diagram of the physical principle of the resonant scattering spectrophotometry shown only on one of the sodium $D$ lines. a) The relative velocity between the Sun-spacecraft is zero. b) There is a non-zero velocity field between them, therefore the solar line is Doppler shifted; this is how the earth-based instruments work. c) A variable magnetic field in the spectrophotometer is placed, enabling 4 sampling points over the solar profile. d) When a quarter wave plate is placed at the GOLF's entrance, it is able to separate the circular polarized components of the solar light

As a consequence, the numerical model simulates the experiment working at ideal conditions at $L_{1}$. It observes the surface of the Sun with the most important and appropriate velocity fields which constitute its velocity background "noise" spectrum. Finally, the simulation of realistic low $\ell$ p-mode and predicted g-mode oscillations also include the true "signal" we are interested in.

\section{Description of the simulation}

The simulation of the experiment has been organized in two main parts. The first one is the simulation of the Sun with all the velocity fields and physical effects that contribute to the solar velocity spectrum, mainly, in the frequency band of interest. The second one is the simulation of the instrumental response from GOLF, including its kinematics respect to the Sun.

In fact, it only consists in calculating what is the intensity measured by our photomultiplier tubes at any time $t$. It has to calculate the convolution between the solar 
sodium $D$ lines and the reference ones resulting from the scattering cross section of the sodium in the vapor cell, shifted by a quantity proportional to the relative line-ofsight velocity. The result is given as the intensities $I_{B, R}^{ \pm}$:

$$
\begin{array}{r}
I_{B, R}^{ \pm}(t)=\iiint I\left(x, y, t, \Delta \lambda-\lambda_{0} V(x, y, t) / c\right) L(x, y) \\
R\left(\Delta \lambda \pm\left(\lambda_{G} \pm \lambda_{B}\right)\right) \mathrm{d} \lambda \mathrm{d} x \mathrm{~d} y(1)
\end{array}
$$

where, $x$ and $y$ are the standard solar disk centered rectangular coordinates, $V(x, y)$ is the velocity component projected onto the line of sight, $c$ the light speed, $\lambda_{0}$ the central frequency of the line, $\Delta \lambda$ is the wavelength relative to the rest wavelength of the solar line, $\pm \lambda_{G}$ is the Zeeman splitting due to the permanent magnetic field, $\pm \lambda_{B}$ is the extra shift due to the small magnetic field provided by a pair of coils, $I\left(x, y, t, \Delta \lambda-\lambda_{0} V(x, y, t) / c\right)$ is the shape of the line at any point in the solar disk at anytime, $L(x, y)$ is the limb darkening, and $R\left(\Delta \lambda \pm\left(\lambda_{G} \pm \lambda_{B}\right)\right)$ is the instrumental response.

Further, the observed velocity by GOLF is the combination of this four intensities in the form of ratios. Amongst others, the classical way (as it is done for actual earth-based instruments) of defining it, would be:

$$
r_{ \pm}=\frac{I_{B}^{ \pm}-I_{R}^{ \pm}}{I_{B}^{ \pm}+I_{R}^{ \pm}}
$$

The selected matrix $(x, y)$ to simulate the solar disk has $128 \times 128$ pixels with a resolution of 15 arcsec per pixel. These values were chosen as a good compromise between the computing time and the spatial resolution needed. However, for some solar phenomena, such as the active regions and the supergranulation, the calculations are done with a higher order matrix and we make a "binning" to reduce the resolution to our standard.

The sodium doublet line profiles have been assumed to be gaussians because the working points of the instrument are close to its core where these are well approximated by a gaussian. For the gaussian solar profiles the parameters introduced for the quiet Sun are a residual intensity of 0.937 and 0.946 and a FWHM of 450 and $650 \mathrm{~mA}$ for the $D_{1}$ and $D_{2}$, respectively (Boumier 1991). On the other hand, it is well known that sunspots and active regions produce changes in the profiles integrated over the solar disk of the solar photospheric absorption lines. In the case of the sodium doublet, lines become broader, shallower and more asymmetric in sunspots (Robillot et al. 1993; Ulrich 1992). These line shapes, when they are added to the quiet Sun line profiles, and integrated over the solar disk, produce changes in the integrated profile that will be measured as a Doppler shift giving rise to "fictitious" velocity fields. To simulate this behaviour, we have used real data of the sunspot positions and areas from the Solar Geophysical Data (Boulder). The software devoped is a generalization of the one built by Jiménez Buendía (1983). It creates a $256 \times 256$ matrix of the Sun with a zero value in the pixels corresponding to the quiet Sun and another one, where there is a sunspot. The appropriate solar line profile can be selected depending if the pixel corresponds to a sunspot or a quiet Sun region. For sunspot areas, we have also kept a gaussian shape with residual intensities of 0.955 and 0.970 and FWHM of 597.6 and $672.4 \mathrm{~mA}$ for both $D_{1}$ and $D_{2}$ lines. Moreover, its continuum is $30 \%$ of the one in the quiet Sun (Régulo et al. 1993). In Fig. 2 we can see the velocity perturbation seen by GOLF for the two considered years. However, there is a problem because only one image of active regions per day is available in the bibliographical records. We have steps of one day in the resultant velocity residuals which have to be corrected for.

As far as the solar continuum intensity hits the cell, it will be only affected by the several filters and optical components that it finds in its way. Changes with time of the properties of these various components will add instrumental noise, but this will not be considered here. However, the change in distance between the spacecraft and the Sun along the year will produce a small modulation of the incident light intensity. In calculating this effect, we found a difference in the incident light of $6.57 \%$.

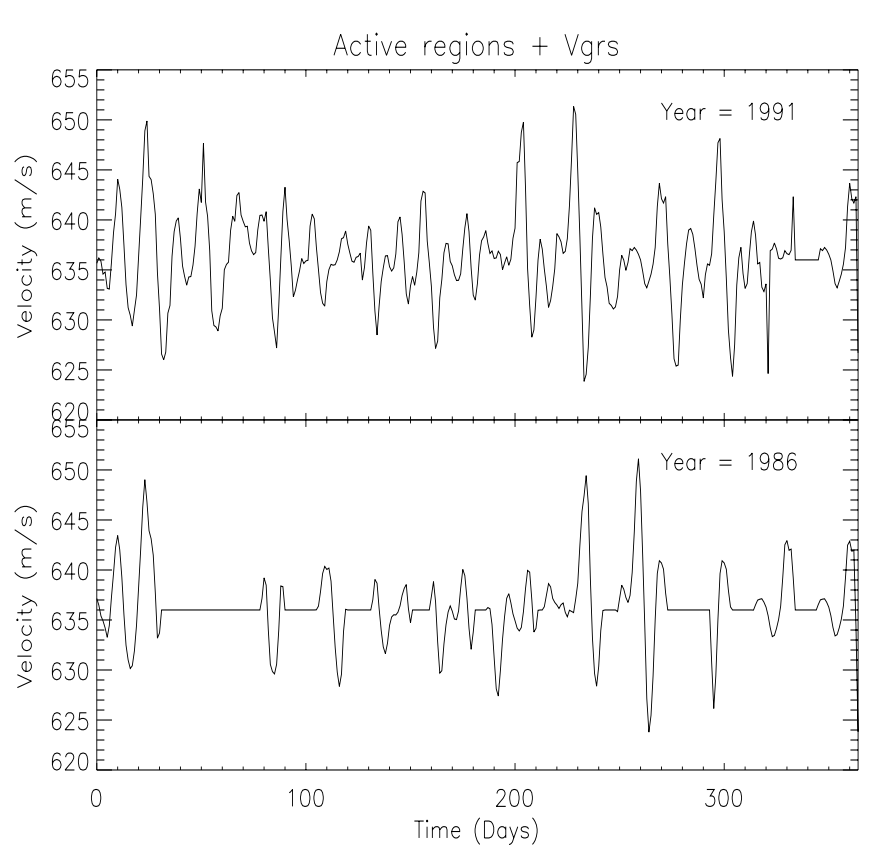

Fig. 2. Velocity perturbation induced by the active regions as they are seen by the GOLF experiment, corresponding to 1991 in the top, and 1986 in the bottom. The average constant velocity corresponds to the velocity offset due to the solar gravitational redshift $\left(V_{\mathrm{grs}}\right)$ 


\subsection{Simulated velocity fields, $V(x, y, t)$}

The velocity fields taken into consideration have been grouped in three categories: the solar "random" velocity fields, $V_{\text {ran }}$, the solar deterministic velocity fields, $V_{\text {det }}$, and the kinematics of the spacecraft relative to the Sun, $V_{\text {kin }}$.

$V(x, y, t)=V_{\operatorname{ran}}(x, y, t)+V_{\operatorname{det}}(x, y, t)+V_{\text {kin }}(x, y, t)$.

\subsection{1. "Random" velocity fields, $V_{\text {ran }}(x, y, t)$}

The "random" simulated velocity fields are the ones related to surface manifestations of the convective zone, such as: supergranulation $V_{\mathrm{sg}}$, giant cells $V_{\mathrm{gc}}$. A meridional circulation $V_{\mathrm{mc}}$, has also been added. Their calculated patterns are refreshed every 30 minutes (much lower than their characteristic time). In this way we have:

$V_{\mathrm{ran}}=V_{\mathrm{sg}}+V_{\mathrm{mc}}+V_{\mathrm{gc}}$.

The most interesting one in our context, is the supergranulation which has the highest contribution to the background velocity spectrum at the periods of hours (Harvey 1985), like the periods predicted for g-modes. There are other two important convective movements namely, the granulation and the mesogranulation. The first one, was not simulated because it influences the solar velocity spectrum at frequencies $\geq 0.5 \mathrm{mHz}$, a region far away from the g-modes. Moreover, its small geometrical scale requires a much higher spatial resolution and, due to its lower characteristic timescale, needs a much lower refreshing time than the others (increasing enormously the amount of computing time). However, a constant effect attributed to it, the velocity limb shift, has been considered and will be described later. The second one, the mesogranulation, was not simulated because it is a velocity field not well studied and its influence in the frequency region of interest for GOLF is believed to be small (Harvey 1985).

To simulate the supergranulation $\left(V_{\mathrm{sg}}\right)$, giant cells $\left(V_{\mathrm{gc}}\right)$ and meridional circulation $\left(V_{\mathrm{mc}}\right)$, a software initially devoped by Hathaway $(1987,1988)$, kindly provided by $\mathrm{F}$. Hill, has been optimized and used. It is based on a decomposition of the velocity field vector over the solar surface in its poloidal and toroidal modes spectrum. The calculated matrix has $256 \times 256$ pixels with a resolution of 7.5 arcsec per pixel. Each point is also subdivided in 4 to increase the accuracy. The selected refreshed time of 30 minutes was chosen as a good compromise between the CPU time spent on each loop and the continuity of the images. The supergranulation is parametrized by an horizontal scale of $30000 \mathrm{~km}$ and a lifetime of 1 day (Anderson \& Avrett 1991). Then, we choose a spectrum of modes between $1 \leq$ $\ell \leq 129$ with $1 \leq|m| \leq \ell$. The individual amplitudes chosen yielded line-of-sight velocities up to $\simeq 410 \mathrm{~ms}^{-1}$. The integration over the solar disk yields residual velocities up to $\simeq \pm 2 \mathrm{~ms}^{-1}$, values that were selected to be consistent with those observed (Pallé et al. 1995). The giant cells are convective structures (not unambiguously detected yet) with predicted sizes of the order of $100000 \mathrm{~km}$ and a lifetime of 30 days. In this case, the modes chosen were sectorial $(m=l)$ with $1 \leq \ell \leq 24$. The integrated residual velocities are in the range of $\simeq \pm 5 \mathrm{~ms}^{-1}$. The selected modes to simulate the meridional circulation were those with $\ell=2$ and $\ell=4$ with $m=0$ and individual velocity amplitudes of $\simeq \pm 5 \mathrm{~ms}^{-1}$. This selection is made on the basis of similarity to what it is observed and/or it is predicted (Hathaway 1988).

\subsubsection{Deterministic velocity fields, $V_{\text {det }}$}

Other solar velocity fields that have been well measured and are of deterministic nature have been also taken into account. These are:

$V_{\mathrm{det}}=V_{\mathrm{rot}}+V_{\mathrm{grs}}+V_{\mathrm{lim}}+V_{\mathrm{osc}}$

where,

$V_{\text {rot }}$, is the projected solar differential rotation, which has been taken from the spectroscopically measured expression (Howard \& Harvey 1970):

$V_{\text {rot }}=\left(\left(-0.308 y^{2}-0.244\right) y^{2}+1.935\right) x$,

$V_{\text {grs }}$, is the gravitational red-shift. It represents the velocity equivalent of the difference of gravitational field between the sodium atoms at the Sun's photosphere and the experiment's cell at $L_{1}$. It has a constant value of $\simeq 633 \mathrm{~ms}^{-1}$ and it is important because it shifts the position on the solar line, where the measurements are made. $V_{\text {limb }}$, is the so-called velocity limb-shift, that appears when the mean line position is measured as a function of position on the Sun. It is found that the convective blue shift decreases from the center towards the limb of the solar disc (Schröter 1957; Beckers \& Nelson 1978). A numerical approximation can be used:

$V_{\operatorname{limb}}=k_{1}\left(1-\sqrt{\left(1-\left(x^{2}+y^{2}\right)\right)}\right)^{k_{2}}$

where $k_{1}$ and $k_{2}$ depend on the observed line. Unfortunately, there is no measured values available for the sodium lines. Instead of that, we have used $k_{1}=125$ and $k_{2}=2$, which are those measured for the potassium resonance line.

$V_{\text {osc }}$, is the solar oscillations velocity contribution. Earthbased solar disk integrated spectrophotometers can measure those modes with $\ell \leq 3$. We have simulated both $\mathrm{p}$ and g-modes up to these degrees. For the acoustic ones, we have introduced 22 different modes with $12 \leq n \leq 33$ for each value of $\ell$, with measured amplitude levs (Régulo, 1987). For the gravity waves, the selected ones were for the $\ell=1,4 \leq n \leq 22$; for $\ell=2,7 \leq n \leq 38$; and for $\ell=3$, $9 \leq n \leq 46$, with $1 \mathrm{mms}^{-1}$ equal amplitude, as predicted by most of the theories. 


\subsubsection{The kinematics, $V_{\text {kin }}(x, y, t)$}

The kinematic effects of the spacecraft can be calculated as:

$V_{\text {kin }}=V_{\text {orb }}+V_{\text {halo }}$

where, $V_{\text {orb }}$, is the radial orbital velocity of the spacecraft at $L_{1}$. It can be calculated as $V_{\text {orb }}=547 \operatorname{sen} \theta$, where $\theta$ is the angle of the Sun-spacecraft vector at any day with respect to its origin at the winter solstice, obtained by inverting the equation:

$$
\frac{t}{365}=\frac{1}{2 \pi}\left(\arccos \left(\frac{e+\cos \theta}{1+e \cos \theta}\right)-\frac{e \sqrt{\left(1-e^{2}\right)} \operatorname{sen} \theta}{1+e \cos \theta}\right)
$$

where $e$ is the eccentricity of the orbit. For this velocity field we calculate one value every day and then we make a linear interpolation to get it anytime during the day.

$V_{\text {halo }}$, is the orbit of the spacecraft around the Lagrangian $L_{1}$ point. The maximum halo velocity is expected to be $\pm 80 \mathrm{~ms}^{-1}$ with a six months period. Therefore we have used a simple sine expression with this period and amplitude.

\subsection{GOLF's cell instrumental response, $R\left(\Delta \lambda \pm\left(\lambda_{G} \pm\right.\right.$ $\left.\left.\lambda_{B}\right)\right)$}

The scattering cross section of the vapor cell will give the reference lines and due to the special magnetic configuration of the instrument, only the $\sigma$ components will be excited giving three groups, $D_{1}, D_{2 a}$ and $D_{2 b}$. Each one has four hyperfine components. The relative heights are in proportion 2:1:3 respectively. The FWHM of the hyperfine components are $\simeq 18.5$ (Boumier 1991). The Zeeman components have been shifted, from its original position, by a quantity $\pm \lambda_{G}$ of $106.1,132.3$ and $79.3 \mathrm{~m} \AA$ due to the permanent longitudinal magnetic field of 5000 Gauss. The separation among the hyperfine components are 5.6, 5.3, $4.8 \mathrm{~m} \AA$, for $D_{1}, D_{2 a}$ and $D_{2 b}$, respectively. Moreover, the extra $\pm \lambda_{B}$ is due to the modulating magnetic field produced by a pair of coils in the pole pieces of the permanent magnet. This has the amplitude of \pm 100 Gauss allowing an instantaneous calibration of the instrument.

\section{The GOLF sensitivity to velocity measurements}

When a celestial rotating body is observed with a high spectroscopic resolution instrument (like GOLF), were this resolution is smaller than the solar linewidth, it is possible to find sensitivity differences over the whole disk (Brookes et al. 1978b). In fact, this can be calculated by instantaneously taking the images of $I_{B}^{ \pm}(x, y)$ and $I_{R}^{ \pm}(x, y)$ by appropriate integration of Eq. (1) at any epoch of the year. Figure 3, shows the result obtained for the sensitivity variation across the solar disk. In the nearest symetrical case, around end September where $V$ is only $\sim 100 \mathrm{~ms}^{-1}$, there is a factor of 4.05 for the red component and 3.85 for the blue one between the maximum and the minimum contributions in the solar disk. In the less symetrical case, around April where $V \sim 1100 \mathrm{~ms}^{-1}$, the factors are 3.96 and 3.73 .

Comparing the sensitivity differences between the two wings we find that it is less than a $7 \%$ and during the year it changes $\sim 2 \%$. Moreover, in April, the reduction of the sensitivity due to the change of the working points is less than a $3 \%$. In conclusion, the selected working points show small differences in their sensibility during the year and between the two wings.

\section{Results and their spectra}

With the software that we have just described we have produced two series of one year long each. The first corresponds to the status of the Sun in 1986, a year of minimum solar activity that it is a good example of what we found in 1996 when GOLF has begun its operations. The other one, is based on the sunspots recorded in 1991 which was a year of a maximum solar activity, which can be considered as a top limit of the activity GOLF is going to face in its lifetime.

The data products of the simulation have been organized in files of one day long. Each one has 1080 blocks of 80 seconds (one GOLF measurement cycle) consisting of one time and 16 intensities. This structure is the same than the one of the real scientific data files.

For each magnetic configuration of the sodium cell $\left(B^{+}\right.$ and $B^{-}$, see Fig. 1) a definition of a "classical" ratio of the mean intensities can be made, similar to the standard two points ratio used by earth-based spectrophotometers:

$$
\begin{aligned}
& r\left(B^{+}\right)=\frac{I_{R}^{+}-I_{B}^{+}}{I_{R}^{+}+I_{B}^{+}} \\
& r\left(B^{-}\right)=\frac{I_{R}^{-}-I_{B}^{-}}{I_{R}^{-}+I_{B}^{-}}
\end{aligned}
$$

Where $I_{B, R}^{ \pm}$, are the intensities directly measured by GOLF (see Fig. 1). An average of the two ratios, $r\left(B^{+}\right)$ and $r\left(B^{-}\right)$, is performed since both have the same behaviour, and the statistical noise will decrease (a comparison with this and other calibration methods can be seen in García et al. 1995). This ratio, $\bar{r}$, is not linearly proportional to the velocity along the year. Therefore, a correction for these non linearities can be empirically calculated following Pallé et al. (1993):

$r^{\prime}=\bar{r}\left(1+c V^{2}+d V^{4}+\ldots\right)$

where $r^{\prime}$ is the linearized ratio and $V$ the radial velocity. To calculate it, we have substituted $V$ by $V^{\prime}\left(V^{\prime}=\right.$ $\left.V_{\text {kin }}+V_{\text {grs }}\right)$, which corresponds to the known velocities and neglecting the other terms which are sufficiently small. 

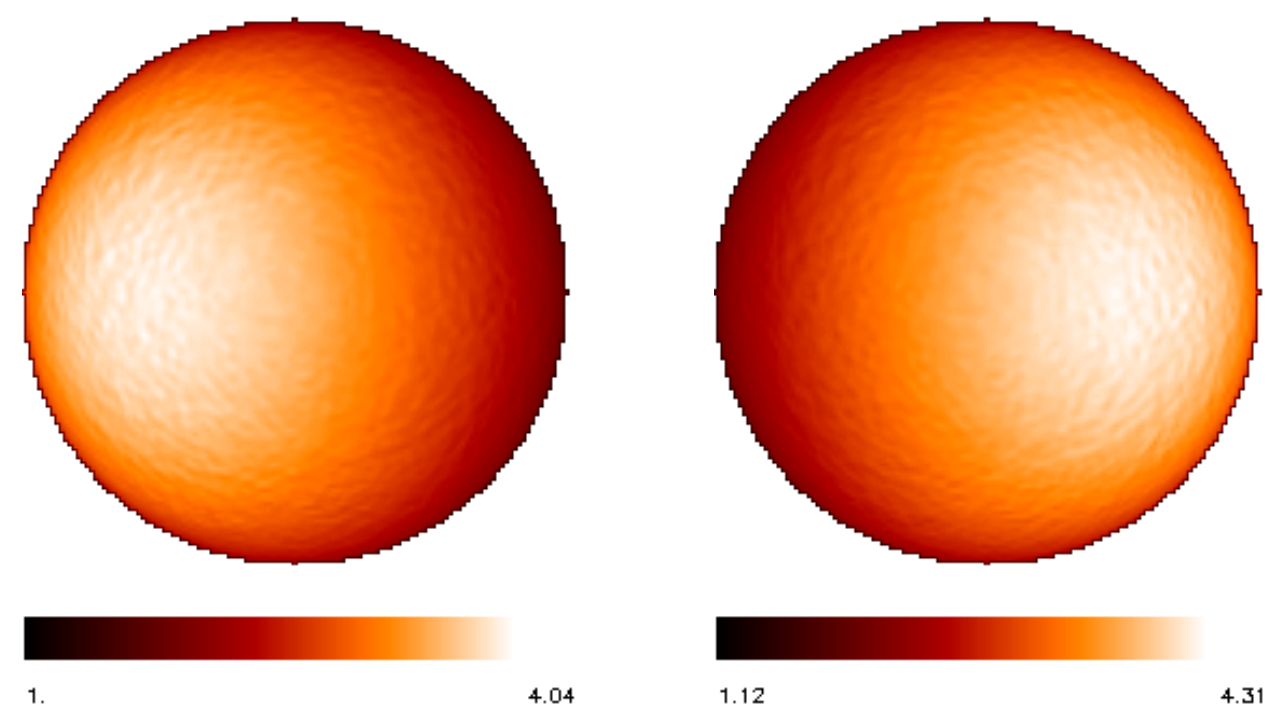

Fig. 3. Maps of the relative contributions of each point over the solar disk to the scattered intensities at each wing of the sodium doublet, $I_{R}^{ \pm}(x, y)$ and $I_{B}^{ \pm}(x, y)$ (left and right respectively) as they are measured by the GOLF instrument. We have considered only the Zeeman components without the magnetic modulation

The calibrated velocity is obtained by a linear fit of $r^{\prime}$ to $\left(a+b V^{\prime}\right)$.

In Table 1 we show the value of each coefficient $(a, b$, $c, d)$ obtained for the whole year. We have also included similar coefficients for the IRIS spectrophotometer (IRIS apparatus in Tenerife only measures the $D_{1}$ component of the sodium doublet).

Table 1. Obtained coefficients of the calibration non linear fitting for the 1991 simulated data series and for one of the earth based IRIS spectrophotometers (Pallé et al. 1993)

\begin{tabular}{cll}
\hline Coefficients & \multicolumn{1}{c}{1991} & IRIS \\
\hline$a$ & $3.436 \pm 0.0053\left(\times 10^{-3}\right)$ & \\
$b(\mathrm{~km} / \mathrm{s})^{-1}$ & $0.20403 \pm 0.00002$ & 0.13 \\
$c(\mathrm{~km} / \mathrm{s})^{-2}$ & $3.78 \pm 0.017\left(\times 10^{-8}\right)$ & 4.5 \\
$d(\mathrm{~km} / \mathrm{s})^{-4}$ & $-9.37 \pm 0.083\left(\times 10^{-15}\right)$ & 2.6 \\
\hline
\end{tabular}

The linealization expression (Eq. 12) has been stopped at the 4 th power of the velocity because it is enough to model the central part of the solar lines where the GOLF working points are located, within the earth based observational errors. On the other hand, we have proved that the more terms we add, the more dependent the coefficients are to the initials values introduced. The last term considered, $V^{4}$, introduces only a small correction to the previous one, $V^{2}$, so the next, $V^{6}$, can be neglected, and the possible corrections would begin to characterize the "noise".

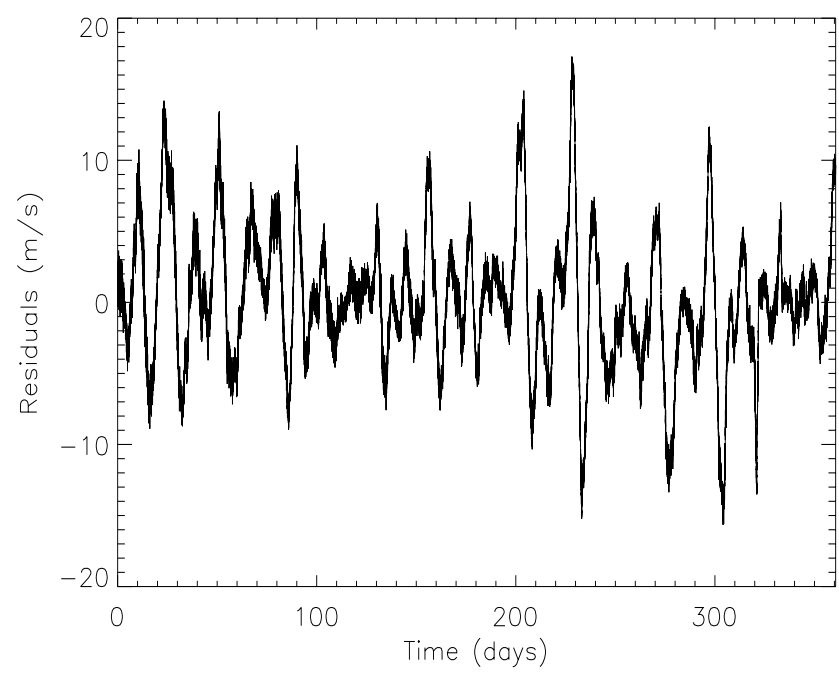

Fig. 4. Example of the simulated velocity residuals obtained for a year with high solar activity

The coefficients are equal in the simulations of both series and there is also a general agreement between the coefficients of the simulation and these from IRIS. The most significative difference is in the $b$ coefficient. This can be explained because GOLF is not only measuring in a different part of the solar line, but also because it measures in the three components of the sodium doublet, which changes significantly the measured slope. There is also the possiblity of small errors due to the not enough precise knowledge of the shape of the Solar sodium lines. A smaller value in $b$ for the IRIS experiments can be 

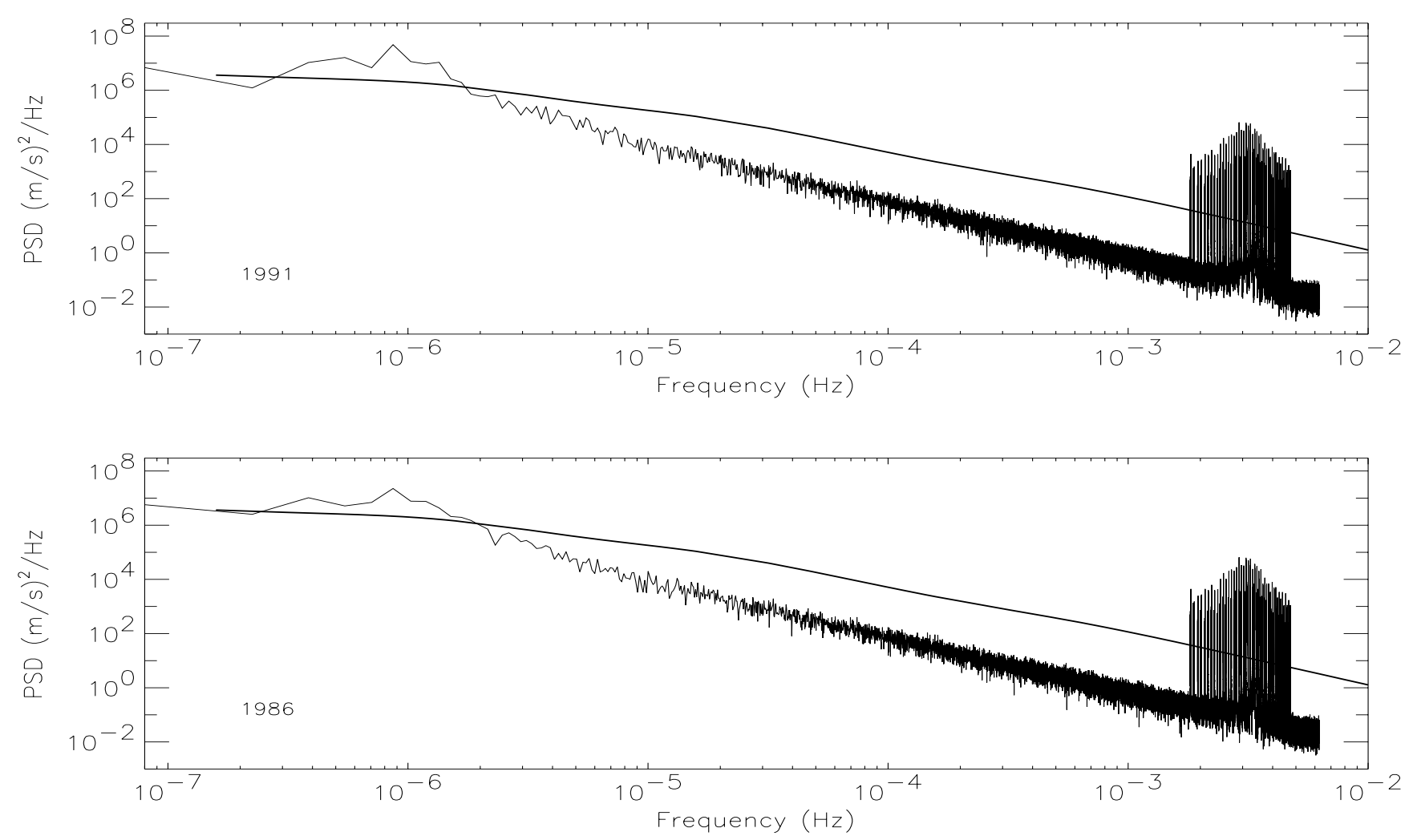

Fig. 5. Power spectral density of the calculated GOLF velocity measurements for high (1991 simulated series) and low (1986) solar activity. It is also shown the best fit to the earth-based observed estimation (Pallé et al. 1995)

explained by an effect of the parasitic light that has not been taken into account in the simulation, giving a higher value of the denominator $\left(I_{R}+I_{B}\right)$ and, therefore, a lower value of $b$.

The velocity residuals $\left(V_{\text {res }}\right)$ are obtained directly substituting the calculated coefficients in the expression:

$V_{\mathrm{res}}=\left(\frac{\bar{r} \cdot\left(1+c V^{\prime 2}+d V^{\prime 4}\right)-a}{b}\right)-V^{\prime}$.

In Fig. 4 we can see the calibrated residuals in velocity. The long time variations correspond to the velocity induced by the active regions passing over the solar disk (compare with Fig. 2), while the small trends are a combination of $V_{\text {ran }}$ and $V_{\text {osc }}$.

\section{Comparison with observations}

To check the validity of the numerical simulation, a comparison of its outputs with the observed background solar velocity spectrum can be performed. Fast Fourier Transforms have been applied to the two simulated series and their power density spectrum calculated. The results are presented in Fig. 5. No significative differences are seen between the two spectra. Only at very low frequencies (below $1 \mu \mathrm{Hz}$ ), where the effects of the active regions are significative, some differencies can be seen.
In both series we have superposed the curve corresponding to the best fitting to the background solar velocity noise spectrum (B.S.V.N.S.) from earth-based observations (Pallé et al. 1995). The power of the simulated series is nearly an order of magnitude lower than the observed one in the region above $100 \mu \mathrm{Hz}$. This difference can be explained mainly due to the earth atmospheric noise, which has not been simulated, and the presence of power due to the window function of the one station observations. However, these effects will not contribute to the observations made in space. Effectively, measurements made by the GOLF experiment in the space (Gabriel et al. 1997) confirm our predictions at low frequencies.

\section{Conclusions}

We have presented a detailed numerical simulation of the observations with GOLF experiment, devoped to check the performances of the instrument which has not been tested with the Sun before the launch. This simulation contains a good representation of the instrumental response, including its kinematics respect to the Sun, and a modelisation of the velocity fields and physical effects that can contribute to the solar velocity spectrum, mainly, in the frequency band of interest for GOLF, from $10 \mu \mathrm{Hz}$ to $6 \mathrm{mHz}$. 
Two data sets, each one year long, have been generated based on how the Sun's activity looks like in 1986, a year of minimum, and 1991, a year of maximum solar activity. With this choice we cover the two extremal values concerning the magnetic activity of the Sun and could be a representation of what GOLF can be facing during its lifetime.

We have computed the theoretical velocity sensitivity of GOLF over the solar disk for the blue and red wing measurements. $\mathrm{A} \sim 7 \%$ of sensitivity difference has been found between the two wings, and seems to be nearly constant for any epoch of the year. In the less favorable case, in April, there is a reduction of the sensitivity less than a $3 \%$, demonstrating that the selected working points are ideal.

We have also applied a calibration of the signal in velocity. We compare the values obtained by this method with the ones for the IRIS instruments showing a general agreement between the two. The main difference found (more than $30 \%$ ) has been with the coefficient leading to the calibration of the velocity signal. This can be explained due to the different slope of the solar lines, where the working points of the two instruments are located (GOLF \& IRIS), and to a higher extent due to the fact that GOLF uses the 3 components of the sodium doublet $\left(D_{1}\right.$ and $\left.D_{2 a, b}\right)$.

The power spectrum of the two data sets available are presented, as well as the best fitted B.S.V.N.S. obtained from the earth-based instruments. The difference of one order of magnitude between the real and the simulated spectra, in the low frequency range of the spectrum, is well understood. The simulation approaches the lev that we have from space-based observations of integral sunlight with the Sun.

Acknowledgements. We wish to thank F. Hill for sending the Hathaway's program. We are also indebted to P. Boumier and P.L. Pallé for their useful comments. This work has been made possible thanks to the financial support of the Spanish CICYT under grants ESP90-0969 and ESP91-1441.

\section{References}

Anderson L.S., Avrett E.H., 1991, En Solar Interior and Atmosphere, Cox A.N., Livingston W.C., Matthews M.S. (eds.). The University of Arizona Press
Beckers J.M., Nelson G.D., 1978, Sol. Phys. 58, 243

Boumier P., 1991, phd Thesis. Université Paris VII

Boumier P., Damé L., 1993, Exp. Astr. 4, 87

Boumier P., van der Raay H.B., Roca Cortés T., 1994, A\&AS 107, 177

Brookes J.R., Isaak G.R., Van der Raay H.B., 1978a, MNRAS 185,1

Brookes J.R., Isaak G.R., Van der Raay H.B., 1978b, MNRAS 185,19

Domingo V., Poland A.I., 1988, The SOHO Mission, ESA SP1104, 5

Fossat E., Roddier F., 1971, Sol. Phys. 12, 352

Gabriel A.H., the GOLF Team, 1995, Solar Phys. 162, 61

Gabriel A.H., Charra J., Grec G., et al., 1997, Solar Phys. (in press)

García R.A., Roca Cortés T., Régulo C., Pallé P.L., 1995, in IV Workshop de SOHO, ESA, SP-376, 2, 369

Grec G., Fossat E., Gelly B., Schmider F.X., 1991, Sol. Phys. 133, 13

Harvey J.W., 1985, in Probing the depths of a Star: the study of solar oscillations from space, Noyes R.W. and Rhodes E.J. Jr. (eds.), JPL 400-327

Hathaway D.H., 1987, Solar Phys. 108, 1

Hathaway D.H., 1988, Solar Phys. 117, 329

Howard R., Harvey J., 1970, Solar Phys. 12, 23

Isaak G.R., Jones A.R., 1988, in IAU Symp. 123, Advances in Helio- and Asteroseismology. S. Frandsen and J. Christensen-Dalsgaard (eds). Dordrecht: Kluwer, p. 467

Jiménez Buendía R., 1983, Tesina. Universidad de La Laguna

Pallé P.L., Fossat E., Régulo C., et al., 1993, A\&A 280, 324

Pallé P.L., Jiménez A., Pérez Hernández F., et al., 1995, ApJ 441,952

Régulo C., 1987, Tesis doctoral, Universidad de La Laguna

Régulo C., Pallé P.L., Roca Cortés T., 1993, in Proceedings of the 5th IRIS workshop and GOLF 93 Meeting, Tenerife, Roca Cortés T. (ed.). IAC: Tenerife

Robillot J.M., Bocchia M., Denis N., 1993, in Proceedings of the 5th IRIS workshop and GOLF 93 Meeting, Tenerife, Roca Cortés T. (ed.). IAC: Tenerife

Schröter E.H., 1957, Z. Astrophys. 41, 141

Snider, J. L., 1970, Sol. Phys. 12, 352

Ulrich R.K., 1992, in GOLF'92 Annual meeting. Paris 